Chimia 45 (1991) 317-318

(C) Schweiz. Chemiker-Verband; ISSN $0009-4293$

\title{
Dyes in Diagnostics
}

\author{
Rupert Herrmann*
}

Abstract. Dyes and dye precursors are often essential components in reagents for medical diagnostics. The type and the attributes of suitable dyes depend on the basis of the technology used for the analysis of the diagnostic parameter to be determined. To avoid interferences by coloured or turbid serum components, dyes which absorb at wavelengths $>550 \mathrm{~nm}$ are preferred. In this article, some diagnostic principles are discussed and examples of applications of dyes are given. Examples demonstrating the versatility of the phenoxazine dye resorufin in the preparation of suitable compounds are also presented.

\section{Introduction}

Diagnostic reagents are an aid for the physician in the diagnosis and treatment of patients. Additionally, they can also be applied in the fields of food and environmental analysis. Dyes and dye precursors are often essential parts of these reagents.

Leucodyes or oxidative coupling systems are used e.g. as chromogenic substrates for peroxidase $/ \mathrm{H}_{2} \mathrm{O}_{2}$ in diagnostics for the determination of metabolites like glucose and cholesterol. The metabolites are measured by the principles of enzymatic analysis in which an intermediate like hydrogen peroxide is generated by a specific and stoichiometric enzyme-catalyzed reaction in a first step. The peroxide formed is used in a second step with peroxidase as catalyst to oxidize the generally colourless chromogenic substrates to coloured compounds whose absorbance can be measured. The sensitivity of such assays strongly depends on the type of chromogenic substrate used.

Similarly, oxidizable metabolites can also be determined via dehydrogenase-catalyzed reaction with the pyridine nucleotide $N A D(P)$ as oxidant. In this case the reductant $\mathrm{NAD}(\mathrm{P}) \mathrm{H}$ is formed. $\mathrm{NAD}(\mathrm{P}) \mathrm{H}$ can be measured in the UV range directly or by diaphorase catalyzed reaction with dye precursors which form dyes through reduction.

Phenolic and anilinic indicator dyes are needed for the preparation of chromogenic and fluorogenic substrates, which can be cleaved by hydrolytic enzymes. With such hydrolase substrates the activity of diagnos- tically relevant enzymes like amylase, lipase or $\gamma$-glutamyltransferase can be measured. Substrates for hydrolases are also important in some sensitive enzyme immunoassays for the detection of hormones, drugs, and proteins, and in methods of nonradioactive nucleic acid diagnostics, which have become very popular in recent years.

Activated fluorescent dyes suitable for coupling to proteins (e.g. antibodies) or lowmolecular weight haptens (e.g. drugs) are required for the synthesis of reagents for fluorescent immunoassays and flow cytometry.

Resorufin and its derivatives are suitable candidates for these applications due to their intensive absorption and emission in the long-wavelength spectral range, which is only slightly disturbed by serum components [1].

\section{Chromogenic Substrates for Redox Enzymes}

Different types of chromogenic substrates for peroxidase $/ \mathrm{H}_{2} \mathrm{O}_{2}$ are described in the literature for enzymatic analysis [2] and nonradioactive immunoassays [3]. For the determination of very low concentrations of $\mathrm{H}_{2} \mathrm{O}_{2}$ or low activities of peroxidase, some sensitive substrates are needed, which can easily be oxidized to dyes with relatively high extinction coefficients. The leuco forms of many dyes could in principle be used for this because peroxidase has relatively low specifity for chromogenic substrates. In practice, however, most of these leucodyes are too easily oxidized by air and, therefore, cannot be used in a marketable product.

To overcome this problem, it is possible to use protected leucodyes, as we have done with the acylated dihydroresorufin derivative shown in the Scheme [4].

The rate of colour formation can to some extent be influenced by the kind of protecting group. The morpholino amide group enhances the water solubility of the substrate. Substrates of this type are prepared by first converting resorufin carboxylic acids to $N, O, O$-triacetylated dihydroresorufin compounds. After conversion to amides or esters, the $O$-acyl groups are selectively cleaved.

Tetrazolium salts are often used as chromogenic substrates for determination of the reductive intermediate $\mathrm{NAD}(\mathrm{P}) \mathrm{H}$ [2]. With the enzyme diaphorase as catalyst, they are reduced by the $\mathrm{NAD}(\mathrm{P}) \mathrm{H}$ into formazan dyes.

The use of resazurin, the $\mathrm{N}$-oxide of resorufin, for measuring $\mathrm{NAD}(\mathrm{P}) \mathrm{H}$ is one of the earliest applications of a resorufin derivative in enzymatic diagnostics [5]. The method is based upon the reduction of the nonfluorescent resazurin to resorufin.

In the field of substrates for redox enzymes, new systems which develop stable colour by reduction and function not only as

Scheme. Acylated Dihydroresorufin Derivative as Substrate for Peroxidase/ $\mathrm{H}_{2} \mathrm{O}_{2}$

*Correspondence: Dr. R. Herrmann

Boehringer Mannheim $\mathrm{GmbH}$

(n) 
Resorufin Derivatives for Fluorescent Labelling of Biomolecules

RESIAC
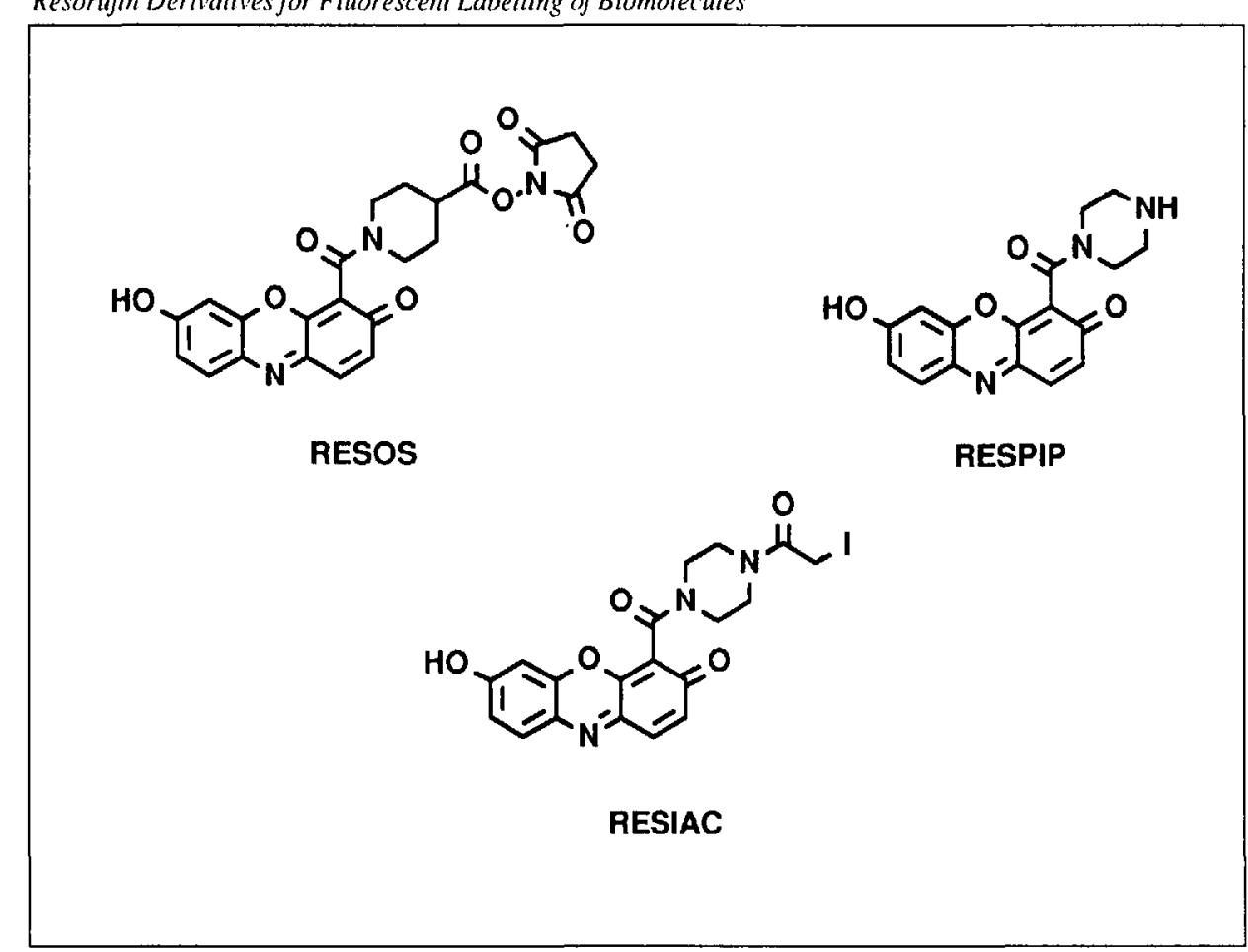

detectable species but also as oxidants with oxidases would be very interesting.

\section{Chromogenic Substrates for Hydrolases}

There are two types of chromogenic substrates known for measuring the activity of hydrolase enzymes: $l$ ) substrates which yield directly measurable dye by action of the enzyme and 2) substrates with which an additional step is necessary after enzymatic cleavage for colour formation.

The normally colourless substrates of the second type are very common as precursors of naturally occurring dyes as e.g. indigo and tyrian purple. Derivatives and analogues of these substrates are often used for visual detection in histochemistry, nucleic acid diagnostics and dry chemistry reagents. The advantages of these substrates include the normally high stability against nonenzymatic cleavage and the definite colour change between substrate and reaction product. The type of additional reaction required depends on the kind of phenolic dye precursor used. Common additional reaction steps are increasing $\mathrm{pH}$, oxidation, oxidative coupling, azo coupling, and complex formation. Oxidation is the additional step with the natural indoxyl substrates and also with substrates made from acylated dihydroresorufins of the type shown in the Scheme [6].

In diagnostic systems where colour is measured continuously by an instrument, it is more convenient to use substrates which develop colour directly when the substrate is cleaved. In this case the phenolic dye must have a $\mathrm{p} K \mathrm{a}$-value lower than the $\mathrm{pH}$ of the solution in which the enzymatic reaction is carried out.
With resorufin and its derivatives it is possible to make a multitude of substrates for measuring the activity of different hydrolytic enzymes such as $O$-dealkylases [7], glycohydrolases [8] and enzymes such as esterases, lipase, phospholipase A2, and cholesterol esterase, which cleave ester bonds [9]. The substrates based on resorufins are not only useful for photometric applications, but also for fluorimetric measurements. They are usually sufficiently stable and allow very sensitive detection of enzyme activity. Resorufin- $\beta$-galactopyranoside, e.g., is one of the most sensitive substrates for the determination of $\beta$-galactosidase activity in enzyme immunoassays [3].

\section{Fluorescent Labels}

For fluorescent labelling of proteins, antibodies, DNA-fragments and other biomolecules, many different activated or functional-group-containing dyes are known [10]. One of the most frequently used dyes for such labels is fluorescein. The disadvantage of fluorescein is its relatively short excitation and emission wavelength, which results in problems from interferences by coloured and fluorescent serum components such as bilirubin. Resorufin, because of its longer excitation and emission wavelength, is much less disturbed by fluorescent serum components than fluorescein. Derivatives of resorufin containing aliphatic amino or carboxyl groups are suitable candidates for the synthesis of fluorescent labels [1].

The $N$-hydroxysuccinimide ester RESOS for example is well suited for fluorescent labelling of biomolecules and low molecular weight compounds. It is sufficiently stable against hydrolysis by water, and reacts specifically with aliphatic amino groups even under neutral conditions. The amide bond formed is more stable than the thiourea bond formed by common isothiocyanate activated labels such as fluorescein isothiocyanate (FITC). The derivative RESPIP is useful for reaction with low-molecular-weight compounds carrying activated carboxylic acid or aldehyde groups. Reaction of RESPIP with iodoacetic acid $N$-hydroxysuccinimide ester yields the fluorescent label RESIAC, which is reactive towards $\mathrm{SH}$-groups.

Conjugates of resorufin with drugs and other low molecular weight compounds of diagnostic interest can be used $e . g$. in sensitive fluorescence immunoassays such as fluorescence polarization immunoassay (FPIA) [11].

\section{Summary}

Dyes and dye precursors play many important roles in diagnostics. The phenoxazin dye resorufin and its derivatives are especially suited for the preparation of various chromogenic and fluorogenic enzyme substrates and fluorescent labels.

I would like to thank my colleagues $H$. Buck, $D$. Freisinger, H.J.Guder, H.-P.Josel, M.Junius-Comer. and $C$. Klein for their contributions to this work.

[1] R. Herrmann, C. Klein, M. Junius-Comer, H.-P. Josel, H.-G. Batz, 'Synthesis and Spectral Properties of New Resorufin Derivatives', in 'Chemistry of Functional Dyes', Eds. Z. Yoshida and T. Kitao, Mita Press, Tokyo, 1988, p. 87-90.

[2] G. Michal, H. Möllering, J. Siedel, 'Chemical Design of Indicator Reactions for the Visible Range', in 'Methods of Enzymatic Analysis, Vol. I, Fundamentals', Ed. H.U. Bergmeyer, Verlag Chemie, Weinheim, 1983, p. 197-232.

[3] B. Porstmann, T. Porstmann, 'Chromogenic Substrates for Enzyme Immunoassays', in 'Nonisotopic Immunoassays", Ed. T.T. Ngo, Plenum Press, New York, 1988, p. 57-84.

[4] Boehringer Mannheim $\mathrm{GmbH}$, EPA 210559 , 1986.

[5] G.G. Guibault, D.N. Kramer, Anal. Chem. 1965. 37,1219

[6] Boehringer Mannhein GmbH, EPA 274700 , 1987.

[7] R.T. Mayer, J.W. Jemyn, M.D. Burke, R.A. Prough, Pestic. Biochem. Physiol 1977, 7, 349.

[8] J. Hofmann, M. Sernetz, Anal. Chim. Acta 1984, 63, 67; Boehringer Mantheim $\mathrm{GmbH}, \mathrm{EPA}$ $156347,1985$.

[9] D.N. Kramer, G.G. Guilbault, Anal.Chem. 1964, 36, 1662; Boehringer Manmheim GmbH, EPA 207252, 1986; EPA 331167 , 1989; EPA 328029 , 1989.

[10] R.P. Haugland, 'Covalent Fluorescent Probes', in 'Excited States of Biopolymers', Ed. R.F. Steiner, Plenum Press, New York, 1983; E. Koller, Appl. Fluoresc. Techinol. 1989, $1,1$.

[11] Boehringer Manmheim GmbH, EPS 209875 , 1990. 